\title{
Analytical Investigation of the Onset of Bifurcation Cascade in Two Logistic-like Maps
}

\author{
S. PANCHEV* \\ Department of Meteorology and Geophysics, Faculty of Physics, University of Sofia, Bulgaria
}

(Received 6 July 2000)

\begin{abstract}
Two modifications of the classical one-dimensional logistic map are proposed, which permitted analytical study of the onset of the bifurcation (period doubling) cascade. The modified maps are two-parametric ones. This introduces new features in their behaviour and makes them more flexible. The results can prove to be useful in the ecological (population dynamics) modeling, where the logistic map is a basic model and also in other fields of application.
\end{abstract}

Keywords: Logistic map; Fixed points; Stability; Bifurcation cascade; Chaos

\section{INTRODUCTION}

The now well-studied logistic map

$$
\begin{gathered}
X_{n+1}=F\left(X_{n}\right)=r X_{n}\left(1-X_{n}\right) \\
X \in[0,1], 0 \leq r \leq 4
\end{gathered}
$$

is one of the most popular "icons" of the nonlinear discrete dynamics (Ott, 1993; Peitgen et al., 1992). Various modifications and generalizations of (1) are known. For example, letting $2 Z=r(1-2 X)$ and $4 C=r(2-r)$, one obtains the equivalent version of (1)

$$
Z_{n+1}=Z_{n}^{2}+C
$$

suitable for extension in the complex plane (Peitgen et al., 1992). Another example is the cubic logistic map (Korsch and Jodle, 1994)

$$
\begin{aligned}
& X_{n+1}=s X_{n}\left(1-X_{n}^{2}\right), \\
& \quad X \in[0,1], 0 \leq s \leq 3 \sqrt{3} / 2 \cong 2.5981
\end{aligned}
$$

This map has one critical point $X_{c}=1 / \sqrt{3}$ and a nontrivial fixed point $\bar{X}_{1}=\sqrt{(r-1) / r}$, which is stable in the interval $1<s<2$. Between 2 and 2.5981 the map develops a bifurcation cascade of period doubling, leading to chaos. Polynomials of higher degree have also been worked out (Sprott, 1993).

\footnotetext{
*e-mail: spanchev@phys.uni-Sofia.bg
} 
Here we study analytically the map

$$
X_{n+1}=F\left(X_{n}\right)=\frac{r}{1+b X_{n}} X_{n}\left(1-X_{n}\right)
$$

where $b>-1,0 \leq r \leq r_{1}(b)$ ( $r_{1}$ to be specified later under the condition $0 \leq X \leq 1)$. To our knowledge, such a map has not been studied before. In Section 2 we show how the properties of the original logistic map (1) change when the new parameter varies. The results can be of interest for some applications. The derivations and discussions go simultaneously. Section 3 deals with an alternative polynomial type version of (4).

\section{ANALYTICAL RESULTS AND DISCUSSIONS}

Simple calculations show that (4) has negative Schwarzian derivative $S_{F}(X, b, r)<0$ and one critical point $\left(F^{\prime}\left(X_{c}, b, r\right)=0\right)$

$$
X_{c}(b)=\frac{1}{b}(-1+\sqrt{1+b})
$$

at which

$$
F\left(X_{c}\right)=G(b) r, G(b)=\frac{1}{b^{2}}(2+b-2 \sqrt{1+b}) .
$$

Obviously $G(0)=1 / 4$ and $0<X_{c}(b) \leq 1 / 2$ for $b \geq 0$, while $1 / 2 \leq X_{c}(b)<1$ for $0 \geq b>-1$. Hence, the value of $r$ at which $F\left(X_{c}\right)=1$ is given by

$$
r_{1}(b)=1 / G(b)
$$

(See Tab. I for $X_{c}$ and $r_{1}$ at $b>0$ and Tab. II at $b<0)$

The new map (4) has two fixed points: $\bar{X}_{0}=0$ for all $r$ and $b$, and

$$
\bar{X}_{1}=\frac{r-1}{r+b} \text { for } r>1, b>-1 .
$$

Since $F^{\prime}(0)=r, \bar{X}_{0}$ is stable (unstable) for $r<1$ $(r>1)$. For the other fixed point

$$
F^{\prime}\left(\bar{X}_{1}\right)=\frac{1}{b+1}\left(2-r+\frac{b}{r}\right), b>-1 .
$$

It is stable if $\left|F^{\prime}\left(\bar{X}_{1}\right)\right|<1$. Hence

$$
1<r<r_{s}(b)=\frac{b+3}{2}+\left[\frac{(b+3)^{2}}{4}+b\right]^{1 / 2}
$$

where $r_{s}$ is the positive root of the equation

$$
r^{2}-(b+3) r-b=0
$$

(See Tabs. I and II for the values of $r_{s}$ ).

For $r>r_{s}$ two additional fixed points $\bar{X}_{2}, \bar{X}_{3}$ appear as roots of the equation $\bar{X}=F(F(\bar{X}))$ with $F(X)$ from (4). However, $\bar{X}_{0}$ and $\bar{X}_{1}$ (already unstable) satisfy this equation too. After factoring

TABLE I Numerical values of $X_{c}, r_{1}$ and $r_{s}$ for $b \geq 0$

\begin{tabular}{llcccccccc}
\hline$b$ & 0 & 0.2 & 0.4 & 0.6 & 0.8 & 0.9 & 0.95 & 0.99 & 1.00 \\
\hline$X_{c}(b)$ & 0.5 & 0.4772 & 0.4580 & 0.4415 & 0.4271 & 0.4204 & 0.4173 & 0.4148 & 0.4142 \\
$r_{1}(b)$ & 4 & 4.3909 & 4.7664 & 5.1298 & 5.4833 & 5.6568 & 5.7411 & 5.8130 & 5.8284 \\
$r_{s}(b)$ & 3 & 3.2613 & 3.5138 & 3.7596 & 4 & 4.1185 & 4.1660 & 4.2244 & 4.2361 \\
$r_{1}-r_{s}=\Delta_{r}$ & 1 & 1.1296 & 1.2526 & 1.3702 & 1.4833 & 1.5383 & 1.5751 & 1.5886 & 1.5923 \\
\hline
\end{tabular}

TABLE II Numerical values of $X_{c}, r_{1}$ and $r_{s}$ for $-1<b \leq 0$

\begin{tabular}{lllllllll}
\hline$b$ & 0 & -0.2 & -0.4 & -0.6 & -0.8 & -0.9 & -0.95 & -0.99 \\
\hline$X_{c}(b)$ & 0.5 & 0.5279 & 0.5635 & 0.6126 & 0.6910 & 0.7597 & 0.8173 & 0.9091 \\
$r_{1}(b)$ & 4 & 3.6036 & 3.1492 & 2.6647 & 2.0944 & 1.7325 & 1.4972 & 1.21 \\
$r_{s}(b)$ & 3 & 2.7266 & 2.4358 & 2.1165 & 1.7403 & 1.5 & 1.3422 & 1.1465 \\
$r_{1}-r_{s}=\Delta_{r}$ & 1 & 0.877 & 0.7134 & 0.5482 & 0.3541 & 0.2325 & 0.155 & 0.0635 \\
\hline
\end{tabular}


out them and solving the resulting quadratic equation

$$
r(b-r) \bar{X}^{2}-(r+1)(b-r) \bar{X}-(r+1)=0
$$

one obtains

$$
\bar{X}_{2,3}(r, b)=\frac{r+1}{2 r} \pm\left[\frac{(r+1)^{2}}{4 r^{2}}-\frac{r+1}{r(r-b)}\right]^{1 / 2} .
$$

The 2-cycle (12) is stable for $r_{s}(b)<r<r_{s 1}(b)$, where $r_{s 1}$ is the smallest positive root of the equation $F^{\prime}\left(\bar{X}_{2}\right) \cdot F^{\prime}\left(\bar{X}_{3}\right)=-1$. However, explicit solution for $r_{s 1}(b)$ cannot be obtained. But $r_{s 1}(0)=$ $1+\sqrt{6}$ as it must be (Hilborn, 1994). Due to the rapidly increasing complexity, further analytical treatment is futureless.

Looking on Table I we see that for $b>0$ the range $\Delta_{r}(b)=r_{1}(b)-r_{s}(b)$ inside which all known bifurcation events (period-doubling cascade, bands of regularity, full chaos) are expected to be observed is wider than $\Delta_{r}(0)=4-3=1$ for the original map (1). In other words, through the new parameter $b(>0)$ one can stretch this range to a desired width. Moreover $r_{1}(b)>4$ and $r_{s}(b)>3$. For various applications where the logistic map serves as a model (ecology, economics, etc.) this can help for better fitting of the model to the data.

An alternative approach to (4) is to fix $r$ and to consider $b$ as a free parameter. With a view to (1), two particular values of $r$ are of special interest $-r=3$ and $r=4$. In the first case $(r=3)$

$$
\bar{X}_{1}=\frac{2}{3+b}, F^{\prime}\left(\bar{X}_{1}\right)=\frac{b-3}{3(b+1)}
$$

Hence, $\left|F^{\prime}\right|>1$ for $-1<b<0$ and $\left|F^{\prime}\right|<1$ for $b>0$. In the second case $(r=4)$

$$
\bar{X}_{1}=\frac{3}{4+b}, F^{\prime}\left(\bar{X}_{1}\right)=\frac{b-8}{4(b+1)}
$$

Hence, $\left|F^{\prime}\right|>1$ (instability) for $-1<b<4 / 5$ and $\left|F^{\prime}\right|<1$ (stability) for $b>4 / 5$.
In the general case, solving (11) with respect to $b$ yields

$$
b_{s}=\frac{r_{s}\left(r_{s}-3\right)}{r_{s}+1}, r_{s} \geq 3
$$

Particularly, for sequence of values $r_{s}^{\prime}=3, r_{s}^{\prime \prime}$, $r_{s}^{\prime \prime \prime}, \ldots, r_{s}^{\infty} \cong 3.57$ where $2^{n}$-cycle $(n=1,2, \ldots)$ first appears in (1), the corresponding sequence $b_{s}^{\prime}=0, b_{s}^{\prime \prime}, b_{s}^{\prime \prime \prime}, \ldots, b_{s}^{\infty} \cong 0.4453$ can be calculated. At $n \rightarrow \infty$ the $b$-sequence obeys the Feigenbaum limit $\delta \cong 4.669 \ldots$, as the $r$-sequence does.

Finally, if $b \equiv r$, i.e.,

$$
X_{n+1}=\frac{r X_{n}\left(1-X_{n}\right)}{1+r X_{n}}, r>1,
$$

then

$$
\bar{X}_{1}=\frac{r-1}{2 r}, F^{\prime}\left(\bar{X}_{1}\right)=\frac{3-r}{1+r}
$$

Hence, $\left|F^{\prime}\right|<1$ for all $r>1$ and $\bar{X}_{1}$ remains a stable point. These are qualitatively new features of the map (4). In this connection, an intriguing question arises related to the modification of (2) on the analogy of (4):

$$
Z_{n+1}=\frac{Z_{n}^{2}+C}{1+b Z_{n}}
$$

where $Z=X+i Y, C=C_{R}+i C_{I}, i=\sqrt{-1}, b$-real parameter. In fact, (17) is a two-dimensional map. At $b=0$ it generates the classical sets of Julia and Mandelbrot (Peitgen et al., 1992). There are three possibilities for the new parameter: $b$-independent, $b \equiv C_{R}, b \equiv C_{I}$. The impact of each one on these sets remains to be investigated numerically.

\section{AN ALTERNATIVE MAP TO (4)}

We now consider the cubic map

$$
\begin{aligned}
X_{n+1}= & F\left(X_{n}\right)=r X_{n}\left(1+a X_{n}\right)\left(1-X_{n}\right) \\
& X \in[0,1],-1 \leq a \leq 1,0<r \leq r_{1}(a),
\end{aligned}
$$

with $r_{1}(a)$ to be defined below $\left(c f . r_{1}(b)\right.$, (7)). Since $(1+b X)^{-1} \approx 1-b X,(18)$ is closely related to (4), 
TABLE III Selected characteristics of the cubic map (18)

\begin{tabular}{lccccc}
\hline$a$ & -1 & $-1 / 2$ & 0 & $1 / 2$ & 1 \\
\hline$X_{c}(a)$ & $1 / 3$ & $(\sqrt{3}-1) / \sqrt{3} \approx 0.4226$ & $1 / 2$ & $(\sqrt{7}-1) / 3 \approx 0.5486$ & $1 / \sqrt{3} \approx 0.5774$ \\
$\bar{X}(a, r)(r>1)$ & $(\sqrt{r}-1) / \sqrt{r}$ & $(3 / 2)-\sqrt{(8+r) / 4 r}$ & $(r-1) / r$ & $(-1 / 2)+\sqrt{(9 r-8) / 4 r}$ & $\sqrt{(r-1) / r}$ \\
$r_{1}(a)$ & $27 / 4=6.75$ & 6 & 4 & 3.1689 & $(3 \sqrt{3} / 2) \approx 2.5981$ \\
$r_{s}(a)$ & 4 & 3.8379 & 3 & 2.4194 & 2 \\
$r_{1}-r_{s}=\Delta_{r}(a)$ & 2.75 & 2.1621 & 1 & 0.7495 & 0.5981 \\
\hline
\end{tabular}

but it may represent independent interest too. Obviously, at $a=1$ one obtains (3), while

$$
X_{n+1}=r X_{n}\left(1-X_{n}\right)^{2} \text { at } a=-1 .
$$

This relationship implies a similarity in some respects in the behaviour of both maps. Actually, let us calculate and compare the same characteristics (5)-(10) for the map (18):

- the critical point and the nonzero fixed point

$$
\begin{aligned}
& X_{c}(a)=\frac{1}{3 a}\left(a-1 \pm \sqrt{a^{2}+a+1}\right) \\
& \bar{X}(a, r)=\frac{a-1}{2 a} \pm \sqrt{\frac{(a-1)^{2}}{4 a^{2}}+\frac{r-1}{a r}}, r>1
\end{aligned}
$$

with $(+)$ at $a>0$ and $(-)$ at $a<0$;

- the stability multiplier

$$
F^{\prime}(\bar{X})=3-2 r-r(a-1) \bar{X}(a, r)
$$

(Unlike (10), the inequality $\left|F^{\prime}\right|<1$ cannot be solved explicitly to obtain $\left.r_{s}(a)\right)$.

- the upper bound $r_{1}(a)$ at which $F\left(X_{c}\right)=1$

$$
r_{1}(a)=3 / X_{c}(a)\left(2+(a-1) X_{c}(a)\right) .
$$

Some particular cases and numerical values of the above characteristics are summarized in Table III. Comparing to Table I and Table II, we observe the same effect of stretching $(a<0)$ or shrinking $(a>0)$ of the first bifurcation range $\Delta_{r}=r_{1}-r_{s}$. Numerically the differences are not essential. However, from technical point of view, the map (4) is easier to work out than (18). In the same time, the extension of (18) in the complex plane

$$
Z_{n+1}=\left(Z_{n}^{2}+C\right)\left(1+a Z_{n}\right)
$$

is simpler than (17) when represented as twodimensional map. Like before, the new parameter $a$ can be free, or $a \equiv C_{R}$, or $a \equiv C_{I}$. The impact of the particular choice on the Julia and Mandelbrot sets remains to be investigated numerically and compared to (17).

\section{CONCLUSION}

In conclusion, we have shown that the proposed modification (4) of the classical logistic map (1) can change the properties of the latter quantitatively (Tabs. I and II) and qualitatively as well. An appropriate choice of $r$ and $b$ can prevent development of bifurcation cascade and chaos. This makes (4) more flexible as a model in ecology (population dynamics). In such a context, (4) can be interpreted as a logistic map with a strengthened $(b>0)$ or weakened $(b<0)$ feedback $\left(1-X_{n}\right)$. Accordingly, $\Delta_{r}(b<0)<\Delta_{r}(0)<\Delta_{r}(b>0)$, where $\Delta_{r}(0)=1$. The same interpretation is valid about (18) and Table III. When compared to each other, the map (4) shows some advantages (deeper and simpler analytical study of the onset of bifurcation cascade). However, (24) is simpler for numerical realization than (17).

\section{Acknowledgment}

This study was supported by the Sofia University Research Foundation under Contract No 268/99. 


\section{References}

Hilborn, R. C. (1194) Chaos and Nonlinear Dynamics. Oxford Univ. Press, p. 654.

Holton, D. and May, R. M. (1993) Models of chaos from natural selection. In: The Nature of Chaos (Mullin, T. Ed.). Clarendon Press, Oxford, p. 314.
Korsch, H. J. and Jodl, H.-J. (1994) CHAOS. A program Collection for the PC. Springer-Verlag, p. 311.

Ott, E. (1993) Chaos in Dynamical Systems. Cambridge Univ. Press, p. 385.

Peitgen, H.-O., Juergens, H. and Saupe, D. (1992) Chaos and Fractals. New Frontier of Science. Springer-Verlag, p. 984. Sprott, J. C. (1993) Strange Attractors. Creating Patterns in Chaos. M\&T Books, p. 426. 


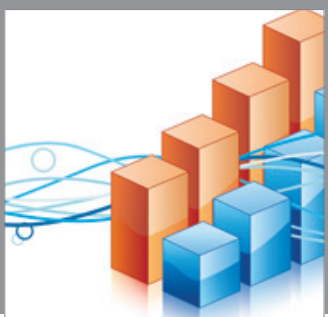

Advances in

Operations Research

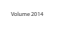

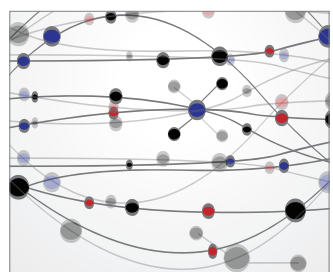

\section{The Scientific} World Journal
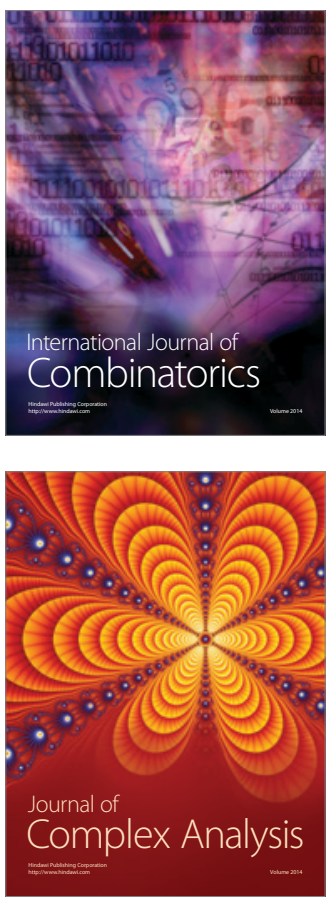

International Journal of

Mathematics and

Mathematical

Sciences
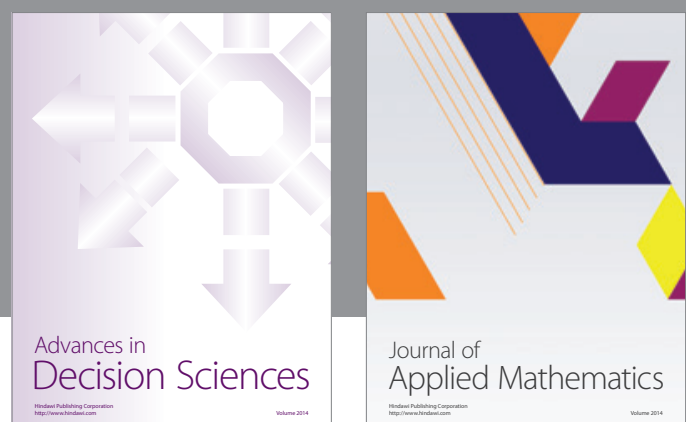

Journal of

Applied Mathematics
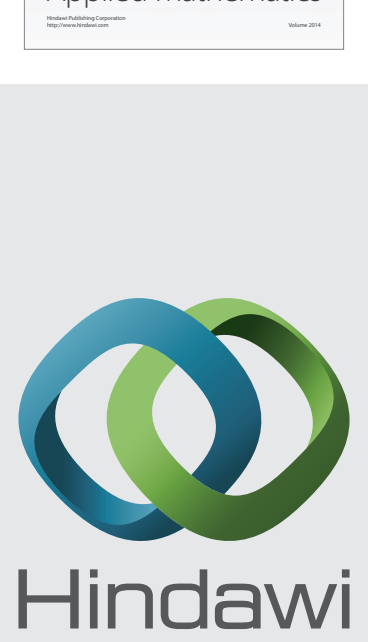

Submit your manuscripts at http://www.hindawi.com
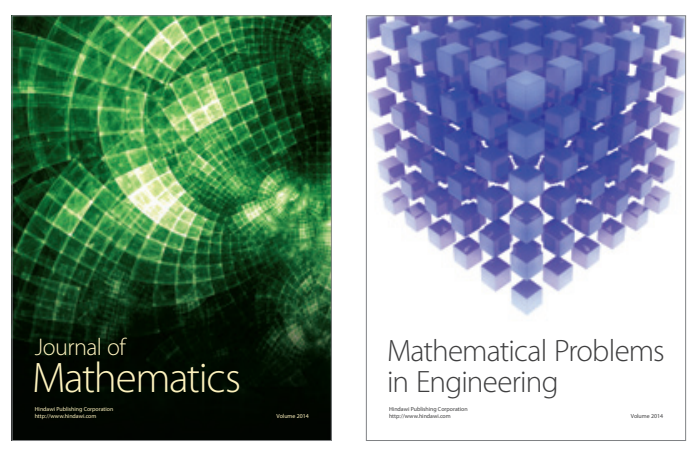

Mathematical Problems in Engineering
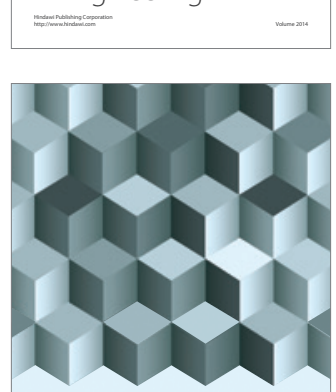

Journal of

Function Spaces
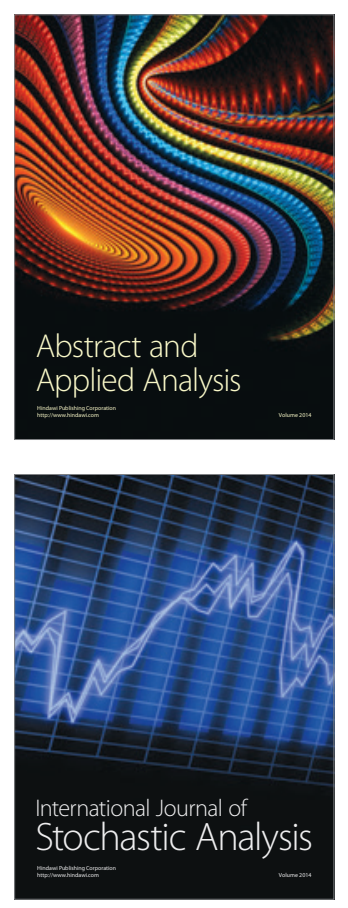

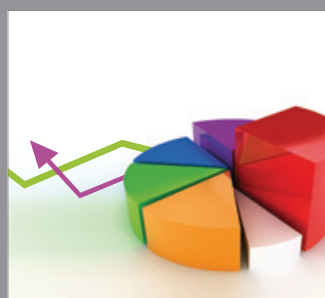

ournal of

Probability and Statistics

Promensencen
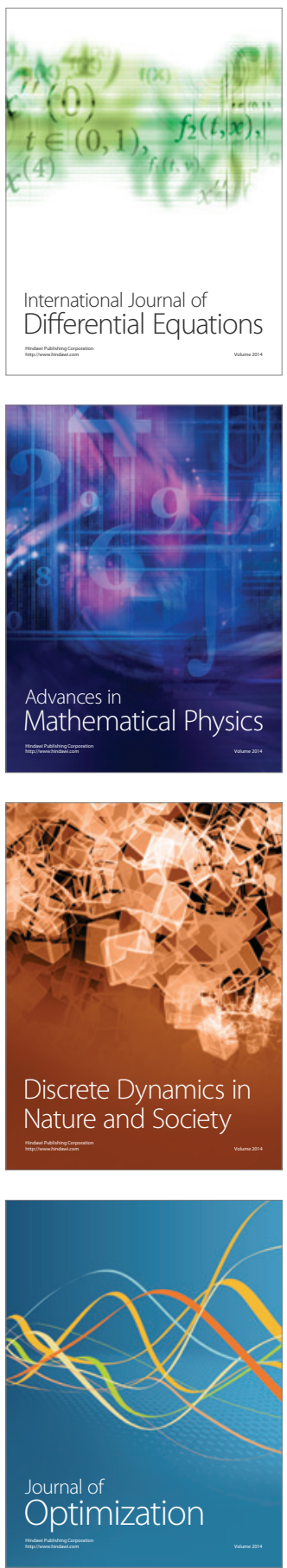